msh-mss Mathématiques et sciences humaines

196 | hiver 2011

Varia

\title{
Trio maudit ou triade féconde ? Le cas du jeu « Pierre, Feuille, Ciseaux »
}

Cursed trio or fruitful triad? The case of the "Rock-Paper-Scissors"

Pierre Parlebas

\section{(2) OpenEdition}

\section{Journals}

Édition électronique

URL : http://journals.openedition.org/msh/12107

DOI : $10.4000 / \mathrm{msh} .12107$

ISSN : 1950-6821

Éditeur

Centre d'analyse et de mathématique sociales de l'EHESS

\section{Édition imprimée}

Date de publication : 30 décembre 2011

Pagination : $5-25$

ISSN : 0987-6936

\section{Référence électronique}

Pierre Parlebas, «Trio maudit ou triade féconde ? Le cas du jeu «Pierre, Feuille, Ciseaux » »,

Mathématiques et sciences humaines [En ligne], 196 | hiver 2011, document 1491, mis en ligne le 15 avril 2012, consulté le 23 juillet 2020. URL : http://journals.openedition.org/msh/12107 ; DOl : https:// doi.org/10.4000/msh.12107 


\title{
TRIO MAUDIT OU TRIADE FÉCONDE ? LE CAS DU JEU «PIERRE-FEUILLE-CISEAUX »
}

\author{
Pierre PARLEBAS ${ }^{1}$
}

C'est un jeu très ancien d'origine incertaine. On compte jusqu'à trois et, à ce moment, les deux joueurs, qui cachaient leur main droite derrière leur dos, la présentent dans un geste symbolique: le poing fermé comme une pierre, ou les doigts réunis ou tendus à plat comme une feuille, ou l'index et le médius écartés comme des lames de ciseaux. La feuille gagne sur la pierre, puisqu'elle l'enveloppe, la pierre gagne sur les ciseaux, puisqu'elle les ébrèche, les ciseaux gagnent sur la feuille, puisqu'ils la coupent.

[Henri Troyat, La pierre, la feuille et les ciseaux]

RÉSUMÉ - Le jeu traditionnel "Pierre-Feuille-Ciseaux», observé dans de nombreux pays, représente un modèle d'interaction de type compétitif que l'on retrouve dans le monde animal et dans certaines situations sociales. S'agit-il, comme on le prétend souvent, d'un modèle triangulaire engendrant une structure cyclique? Sa structure sous-jacente est en réalité celle d'un jeu à deux joueurs et à somme nulle (ou «duel»), alors que celle d'un autre jeu «Renard-Poule-Vipère », apparemment isomorphe, dénote effectivement une configuration triadique circulaire d'un autre type. L'intransitivité suscite un paradoxe caractérisé par une ambivalence créatrice d'ambigüité entre les interactions d'opposition et de coopération.

La triade en général est revendiquée comme l'unité relationnelle fondamentale par de nombreux sociologues. Mais la triade paradoxale, qui provoque des effets pervers, est rejetée par les institutions notamment par les instances sportives, alors qu'elle semble féconde et favorable à l'épanouissement d'un lien social, facteur d'ouverture et d'adaptabilité.

MOTS-CLÉS - Duel, Effet Condorcet, Graphes équilibrés, Intransitivité, Jeu paradoxal, Jeu traditionnel, Tournoi, Triade

SUMMARY - Cursed trio or fruitful triad? The case of the "Rock-Paper-Scissors" Game Observed in many countries, the "Rock-Paper-Scissors" traditional game represents a competitive interaction model we also find in the animal world and in some social situations. Is it, as is often claimed, a triangular model engendering a cyclic structure? Its underlying structure is in fact the one of a game with two players and a zero-sum, whereas the "Fox-Chicken-Snake” game's structure, apparently isomorphous, denotes in fact a circular triadic configuration. This intransitivity generates a paradox characterized by an ambivalence which creates ambiguity between opposition and cooperation interactions.

Many sociologists hold triads to be the fundamental relational unit. But paradoxical triads, which have perverse effects, are rejected by institutions, especially sport institutions, even though they seem fruitful and favourable to the blossoming of a social link, a factor of open-mindedness and adaptability.

KEYWORDS - Balanced graphs, Intransitivity, Paradoxical game, Traditional game, Tournament, Triad, Voting paradox, Zero sum two persons game

\footnotetext{
${ }^{1}$ Laboratoire de sociologie GEPECS (Groupe d'Étude pour l'Europe de la Culture et de la Solidarité, EA 3625), Faculté des sciences humaines et sociales - Université Paris Descartes-Sorbonne, 12 rue Cujas, 75230 Paris cedex 05,pparlebas@free.fr
} 
Certains jeux traditionnels présentent d'étonnantes similitudes avec des situations d'interaction du monde animal. Ainsi en est-il des jeux tels «Pierre-Feuille-Ciseaux » ou « Renard-Poule-Vipère » qui mettent en opposition trois symboles selon une relation circulaire déconcertante au cours de laquelle chaque symbole en domine un autre et est dominé à son tour par le troisième. Des éthologues en ont identifié la fidèle réplique dans les situations de domination qui caractérisent certaines populations animales, notamment chez le lézard californien étudié par B. Siverno et C.M. Lively. Soulignant cette analogie insolite, l'informaticien Jean-Paul Delahaye analyse de façon détaillée des simulations informatiques de grande ampleur qui ont exploré les ressources de cette circularité des dominances [Delahaye, 2008]. La conclusion qu'il tire de l'évolution des modèles étudiés est riche de conséquences : "Les relations de domination cyclique entre souches ou espèces dans le monde vivant, affirme-t-il, sont l'une des causes du maintien de la diversité biologique ».

Ce chercheur n'hésite pas à pousser la comparaison à l'extrême en avançant que «Le cas du lézard californien Uta stansburiana est frappant, car trois variétés de lézards semblent y jouer à Pierre-Feuille-Ciseaux ». Le propos de J.-P. Delahaye est original et stimulant; il invite à examiner de plus près les caractéristiques du jeu de référence et à essayer de saisir quels types d'effets la relation circulaire de dominance entraîne sur le comportement des différents participants. «Les mathématiciens, écrit cet informaticien, ont transformé un jeu d'enfants, Pierre-Feuille-Ciseaux, en un riche modèle des interactions animales ». Comment se présente donc la structure de ce jeu ? La retrouvet-on dans d'autres jeux et avec les mêmes propriétés ? Quelles sont les implications relationnelles, négatives ou positives, que cette configuration provoque chez l'ensemble des joueurs?

\section{LE TRIO INSOLITE DE BASE}

Le jeu «Pierre-Feuille-Ciseaux » est très ancien, indique J.P. Delahaye ; on le retrouve en Chine, au Japon, aux Philippines, en Corée ou en Hongrie, assorti de trois figures emblématiques diverses, telles «Guerrier-Tigre-Mère du guerrier» ou "SerpentGrenouille-Limace»(encore des animaux !). On sait que dans ce jeu, au signal de départ, chacun des deux joueurs, face à face, allonge un bras dont la main figure l'un des trois symboles-phares, sachant que la Pierre casse les Ciseaux, que les Ciseaux coupent la Feuille et que la Feuille recouvre la Pierre. La matrice de domination des trois symboles et son graphe correspondant représentent la structure élémentaire de cette configuration basique (cf. Figure. 1).

Le jeu «Renard-Poule-Vipère », encore appelé « les Trois camps », repose sur la même structure cyclique de domination; dans cette activité ludique, les joueurs sont disposés sur le terrain dans trois camps antagonistes dont les pouvoirs de prise respectifs forment une boucle: les Renards capturent les Poules, les Poules s'emparent des Vipères, et les Vipères dominent les Renards (cf. Figure 2).

La configuration reliant les trois espèces de lézards évoquées précédemment offre, elle aussi, un réseau cyclique analogue. Par l'intermédiaire des femelles, les mâles à gorge Orange dominent les mâles à gorge Bleue, ceux-ci supplantent les mâles à bandes Jaunes, et ces derniers l'emportent à leur tour sur les mâles à gorge Orange : là encore, le chemin de dominance se ferme en un circuit. 


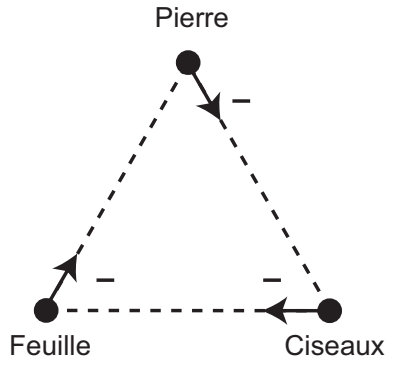

$\longrightarrow--$ Relation de domination

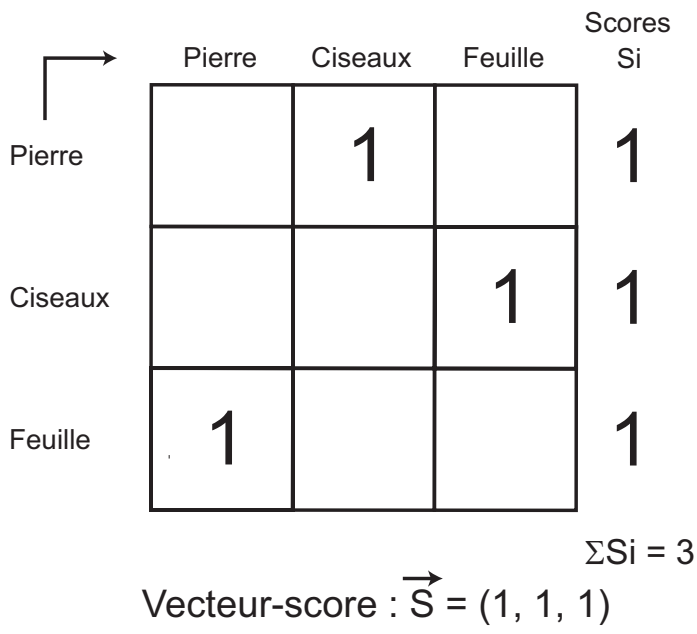

FIGURE 1. Relation circulaire de domination du jeu «Pierre-Feuille-Ciseaux » La relation est intransitive

Le constat est immédiat. Ces trois situations sont isomorphes; elles peuvent être représentées par la même matrice et le même graphe de domination auxquels est associée une propriété insolite : le «dernier» des trois sujets domine toujours le «premier » (cf. Figure 2)!
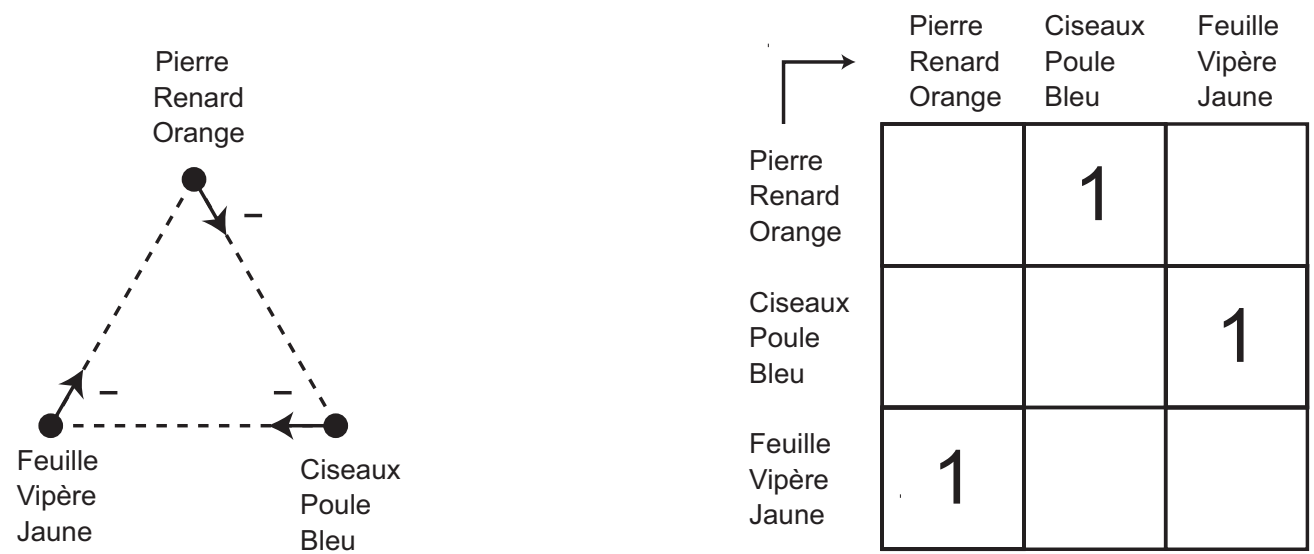

$\longrightarrow$ - - Relation de domination

FIGURE 2. Graphe et matrice de domination communs aux trois situations d'interaction

Cette isomorphie des trois modèles indique-t-elle l'identité fondamentale des trois situations ?

Devant une telle similitude, on est tenté de considérer que ces trois situations d'interaction possèdent la même structure fondamentale. Cependant, comme nous le verrons, l'observation des comportements de terrain tend à remettre cette opinion en cause et invite alors à approfondir les caractéristiques de la relation de dominance. En effet, «Pierre-Feuille-Ciseaux » se déroule dans une atmosphère de rivalité serrée mais dénuée de toute confusion quant aux issues des affrontements clairement tranchées (quand les deux symboles présentés sont identiques, le coup est simplement annulé). En revanche, à «Renard-Poule-Vipère », on se retrouve dans la cour du roi Pétaud: les joueurs semblent désorientés, car faisant tout ce qu'il faut pour gagner, ils s'enfoncent 
dans la défaite! Ainsi, les Renards qui ont capturé de nombreuses Poules, se réjouissent-ils dans un premier temps, avant de s'apercevoir qu'en éliminant ces Poules, ils viennent de se priver de leur seul protecteur à l'égard des Vipères qui les menacent directement. Pour chaque joueur, et pour chacune des trois équipes, tout résultat semblant favoriser leur réussite se transforme en une reculade vers l'échec.

Comment rendre compte de cette flagrante disparité entre les comportements engendrés par deux jeux dont les structures ludiques fondamentales respectives sont en apparence rigoureusement identiques?

\section{RELATION DE TOURNOI ET EFFET CONDORCET}

La relation de domination $\mathrm{R}$ qui, dans les situations précédentes, porte seulement sur trois éléments peut, bien entendu, s'adresser à des effectifs de symboles plus importants. En France, par exemple, il est courant d'ajouter à « Pierre-Feuille-Ciseaux » un élément supplémentaire, le «Puits» $(T)$ qui absorbe la Pierre et les Ciseaux mais qui est recouvert par la Feuille (ce qui confère au Puits et à la Feuille un pouvoir supérieur à celui des deux autres symboles (cf. Figure 3 ).

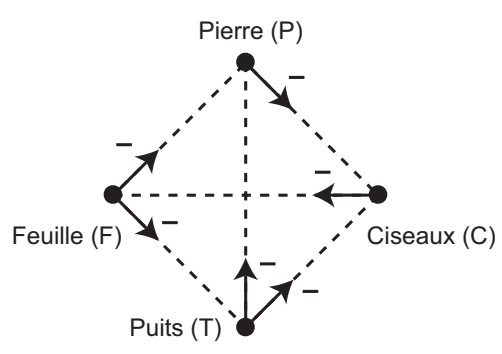

$\longrightarrow$ - - - - Relation de domination

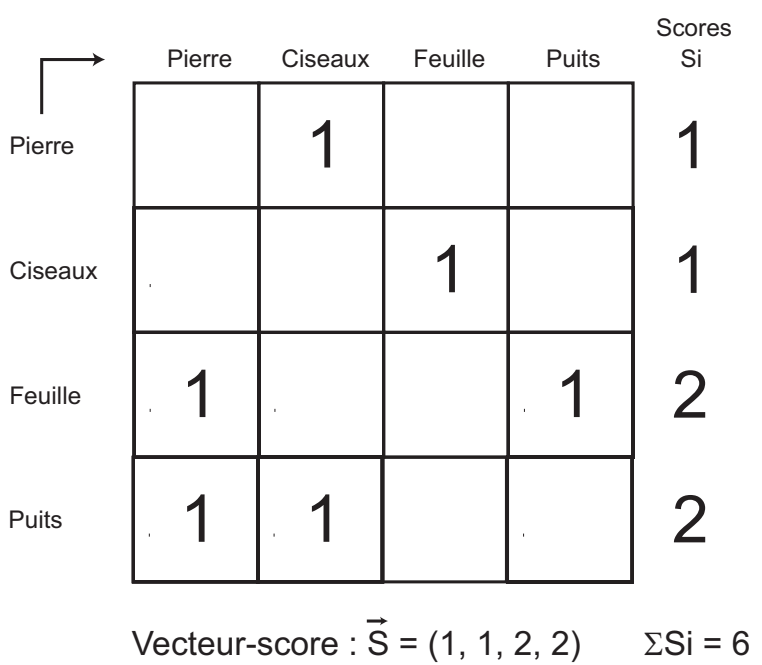

FIGURE 3. Le jeu « Pierre-Feuille-Ciseaux » auquel on a ajouté le Puits Ce graphe de tournoi possède un degré maximal d'intransitivité: un circuit hamiltonien $(\mathrm{P}, \mathrm{C}, \mathrm{F}, \mathrm{T}, \mathrm{P})$ et deux triplets intransitifs $(\mathrm{P}, \mathrm{C}, \mathrm{F}, \mathrm{P})$ et $(\mathrm{C}, \mathrm{F}, \mathrm{T}, \mathrm{C})$

\subsection{INTRANSITIVITÉ ET EFFET CONDORCET}

Les caractéristiques techniques de la relation de domination $\mathrm{R}$ ont donné lieu à une foule de travaux et ont été développées dans des champs d'application variables par un grand nombre de mathématiciens: K. Arrow, G.-Th. Guilbaud, F. Harary, M.G. Kendall, B. Monjardet, O. Hudry... Dès le XVIII ${ }^{\mathrm{e}}$ siècle, dans sa recherche des meilleures règles électorales possibles pour rendre compte de la volonté générale, l'encyclopédiste Condorcet avait proposé de traiter, les unes après les autres, toutes les paires de «candidats » en lice, puis de composer ces préférences binaires selon la règle majoritaire. L'opinion collective résultante, a-t-il montré, pouvait alors être incohérente; des opinions individuelles rationnelles peuvent engendrer un « paradoxe », c'est-à-dire une opinion collective intransitive, irrationnelle : si le candidat $A$ est préféré au candidat $B$, si $B$ est préféré à $C$, et si $C$ est préféré à $\mathrm{A}$, alors on obtient une opinion 
circulaire, un paradoxe indécidable [Condorcet, 1785]. L'intérêt suscité par cette procédure a conduit les chercheurs en sciences sociales à en généraliser l'usage à une « opinion» purement individuelle : la composition des choix binaires émis par un «votant» isolé donne lieu à une opinion susceptible, elle aussi, d'être affectée d'intransitivité (cette caractéristique a été fréquemment utilisée dans les enquêtes en sciences humaines et sociales). Or, cette structure "paradoxale », c'est celle que nous avons observée dans les trois situations précédentes de jeux et d'interactions animales.

Quand elle s'applique sur toutes les paires des sommets considérés, cette relation binaire orientée de domination $R$ - qui représente le fil rouge de cette étude - a été nommée «relation de tournoi » par les chercheurs, précisément par analogie avec les tournois des jeux sportifs. S'exerçant sur un ensemble $E$ d'éléments, elle est caractérisée par les propriétés suivantes :

- La relation est totale :

$$
\forall x, y \in E: \quad(x, y) \notin R \quad \Rightarrow \quad(y, x) \in R
$$

- La relation est antisymétrique :

$$
\forall x, y \in E: \quad(x, y) \in \mathrm{R} \quad \Rightarrow \quad(y, x) \notin R
$$

- La relation peut être, ou ne pas être, transitive

- La relation est transitive :

$$
\forall x, y, z \in E: \quad(x, y) \in R \text { et }(y, z) \in R \quad \Rightarrow \quad(x, z) \in R
$$

- Sinon, la relation est intransitive : au moins un triplet est un circuit.

Une relation de tournoi correspond donc à une relation orientée binaire, complète et antisymétrique. Si elle est transitive, elle définit une relation d'ordre total sur les sujets observés, c'est-à-dire un classement qui hiérarchise tous les éléments sur une « échelle», sans anicroche. Si elle est intransitive, l'ordre est transgressé ; un ou plusieurs cycles apparaissent, introduisant dans le classement, des contradictions qui offusquent la logique traditionnelle. C'est la présence de ces cas d'intransitivité, affectant la relation de tournoi, que G.-Th. Guilbaud a nommée «effet Condorcet » [Guilbaud, 1952, 1968]. Cet effet est plus ou moins intense ; il peut provoquer la surgie d'un nombre variable de triplets intransitifs et solliciter même éventuellement tous les sommets du graphe sur un circuit dit «hamiltonien» (tournoi «irréductible») (cf. Figure 3). Des cas d'intransitivité peuvent, bien entendu, surgir au sein de relations qui ne seraient pas des tournois ; cependant, le chercheur a intérêt à concevoir et à traiter ses données sous forme de tournoi, quand cela est possible, afin de s'appuyer sur des propriétés plus richement exploitables.

Le noyau basique de l'effet Condorcet est un triplet : il y faut nécessairement trois éléments. La dyade n'y suffit pas. Seule, la présence d'un troisième sommet peut faire surgir une éventuelle intransitivité, qui est le cœur du problème. Autrement dit, une relation binaire peut provoquer des conséquences paradoxales mais seulement par une prise en compte ternaire des éléments : il y a changement de niveau. Sans doute cette caractéristique joue-t-elle un rôle non négligeable dans l'apparition du paradoxe et dans son interprétation par les acteurs sur le terrain.

\subsection{L’EFFET CONDORCET EN SOCIOLOGIE ET EN ÉTHOLOGIE}

Habituellement, en ce domaine, le chercheur recueille, après enquêtes, des données comparatives qui correspondent à des préférences ou à des dominations par paires qu'il traite sur le plan individuel ou sur le plan de la préférence collective majoritaire 
résultante. Il observe alors si la composition de ces données produit un ordre total ou, éventuellement, des incohérences sous forme d'effet Condorcet qu'il devra interpréter. Dans le cas des jeux ici étudiés, c'est en quelque sorte une démarche inverse qui est proposée : l'effet Condorcet est provoqué d'emblée par la règle ; le chercheur doit observer et analyser les différents types de comportements qui en découlent, pour finalement leur attribuer une signification généralisable.

Abondamment explorée par les mathématiciens, la relation de tournoi a donné lieu à de nombreux travaux sociologiques et éthologiques. La hiérarchie qui s'établit au sein des groupes d'animaux détermine un tournoi, parfois affecté d'intransitivité (ce qui peut être d'ailleurs à la source de l'évolution de cette hiérarchie). En s'appuyant sur des travaux d'éthologie de Hall et De Vore, Théodore Caplow a dressé les matrices de dominance fondées sur les résultats majoritaires des combats « un contre un », observés au sein de groupes de babouins [Caplow, 1971]. Il obtient un graphe de tournoi qui définit fréquemment une hiérarchie linéaire dénuée d'intransitivité, c'est-à-dire un ordre total.

Cette relation de subordination animale est souvent stable; elle revêt une grande importance car elle assure la régulation de la vie sociale des sociétés considérées. «Les rapports de dominance et de hiérarchie contribuent, pour l'essentiel », écrit l'éthologue Jacques Goldberg, "à la constitution de l'organisation et des structures des sociétés » [Goldberg, 1998]. Quand un animal, unique, arrive en tête de cette hiérarchie en dominant tous les autres, il est appelé l'animal «alpha»par les éthologues et est nommé le «vainqueur de Condorcet » par les mathématiciens (qu'il y ait ou non des cas d'intransitivité parmi les autres). Souvent, on observe des conflits subalternes, échelonnés sur l'échelle de dominance, qui suscitent des circuits entre animaux de niveau inférieur sans affecter l'animal alpha (circuit de type I). La rivalité prend une dimension plus décisive quand elle met en circuit l'animal alpha avec ses suivants immédiats, «bêta» et «gamma». Le tournoi est alors étêté (circuit de type II) et le groupe risque d'en être désorganisé.

Dans le domaine sociologique, après les travaux de Borda et de Condorcet, dans un premier temps c'est le champ électoral qui a suscité une avalanche de travaux de la part des chercheurs [Monjardet, 1973 ; Monjardet et Hudry, 2003]. Puis, notamment par le recours à la méthode des comparaisons par paires, la relation de tournoi a trouvé de nombreux domaines d'application liés à des questions d'évaluation, de choix professionnels, de goûts esthétiques ou de préférences relationnelles, tant au niveau individuel que collectif. Dans le champ des préférences électorales et sociales, G.-Th. Guilbaud a ouvert une piste féconde en considérant que l'effet Condorcet représente le «symptôme » d'une «profonde division sociale » [Guilbaud, 1968]. Ainsi envisagé, le paradoxe devient le révélateur d'un conflit sous-jacent et un outil d'auscultation des cohésions de groupes, sachant que la cohérence cognitive peut être en relation avec la cohésion affective et groupale [Parlebas, 1971, 1972].

On sait qu'en 1951, Kenneth J. Arrow a généralisé le cas de l'effet Condorcet en démontrant que cette incohérence logique provoquait l'impossibilité de mettre au point une procédure de choix, et notamment une règle électorale qui respecterait les propriétés de logique élémentaire, exigibles et acceptables dans toute démocratie [Arrow, 1951]. Cette conclusion ne nie évidemment pas la réalité des démocraties mais dévoile des obstacles inévitables et met sur la voie de solutions électorales affaiblies et raisonnables de contournement [Barbut, 1959 ; Hudry, 2003].

Ces quelques remarques révèlent que les particularités du jeu «Pierre-FeuilleCiseaux » apparemment anodin, représentent en réalité la configuration canonique 
située au centre d'une myriade de situations éthologiques et sociologiques de grand intérêt, dont les aspects interdisciplinaires ne sont pas négligeables.

\section{DU TRIO AU DUO}

Dans la réalité des phénomènes sociaux, la présence d'un triplet paradoxal qui défie la rationalité, incite à réinterpréter ou à réorganiser la situation afin de s'acheminer vers une solution logiquement satisfaisante. Comment faire disparaître le paradoxe aux connotations souvent subversives ? Plusieurs solutions intéressantes, adaptées à leur contexte, ont été proposées.

\subsection{UNE SITUATION SOCIO-POLITIQUE : L’ÉLECTION PRÉSIDENTIELLE FRANÇAISE}

Dans son analyse de l'élection présidentielle française de 1974, Pierre Batteau met en évidence comment la présence subreptice d'un effet Condorcet opportunément décelé, va déclencher une stratégie offensive, apparemment iconoclaste, de la part d'un haut responsable politique [Batteau, 1979].

Les sondages d'opinion préalables à l'élection, avaient révélé que les intentions de vote réparties sur les trois candidats les plus en vue, dénotaient des pourcentages de voix tranchés qui classaient en tête François Mitterrand (45\% des voix), Jacques Chaban-Delmas en second $(25 \%)$ et Valéry Giscard d'Estaing en troisième position $(18 \%)$. Une nouvelle enquête plus fine révélait que les choix émis en situation de comparaisons par paires accordaient la préférence à $\mathrm{F}$. Mitterrand sur J. Chaban-Delmas (57 \%), à J. Chaban-Delmas sur V. Giscard d'Estaing (69 \%) et à V. Giscard d'Estaing sur F. Mitterrand (51\%) (cf. Figure 4). On était donc en présence d'un graphe cyclique révélateur d'un effet Condorcet, identique au modèle triangulaire des jeux précédemment examinés. Dans le scrutin uninominal majoritaire à deux tours qui définit cette élection présidentielle, le résultat final semblait prévisible : le premier tour allait laisser face à face dans l'arène F. Mitterrand et J. Chaban-Delmas, et le second tour consacrerait la victoire de F. Mitterrand.

\begin{tabular}{|c|c|}
\hline $\begin{array}{c}\text { Comparaisons } \\
\text { par paires }\end{array}$ & $\begin{array}{c}\text { Majorité } \\
\text { favorable }\end{array}$ \\
\hline F. M. > J. Ch.- D. & $57 \%$ \\
\hline J. Ch.- D. > V. G. d'E. & $69 \%$ \\
\hline V. G. d'E. > F. M. & $51 \%$ \\
\hline
\end{tabular}

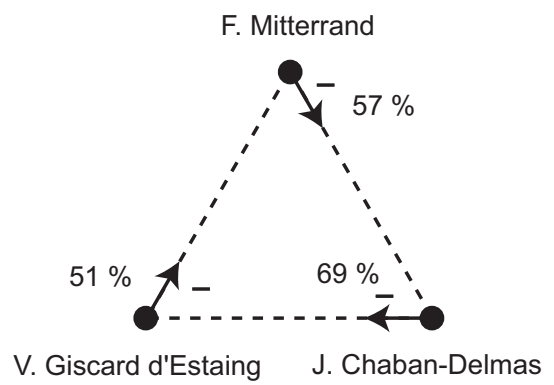

FIGURE 4. État de l'opinion avant l'élection présidentielle française de 1974 La triade de tête des candidats présente un effet Condorcet insolite : aucun des candidats ne domine les deux autres ; chacun en domine un seul et est dominé par le troisième

L'effet Condorcet allait être exploité de façon quelque peu machiavélique. Le ministre de l'Intérieur, Jacques Chirac, mobilisa résolument les représentants de la majorité de l'époque afin de provoquer l'abandon de leur candidat «naturel» (J. Chaban-Delmas) au profit d'un allié beaucoup moins prisé (V. Giscard d'Estaing) mais seul susceptible de devancer leur adversaire (F. Mitterrand) dans le duel décisif du second tour. Ainsi fut fait. Le candidat V. Giscard d'Estaing fut élu Président par une 
faible majorité $(50,3 \%$ ) (et $\mathrm{J}$. Chirac fut nommé Premier Ministre). En réussissant à imposer la paire F. Mitterrand/V. Giscard d'Estaing, au second tour prévu par le règlement, J. Chirac brisa l'effet Condorcet, mais en sacrifiant le candidat « historique » de son parti.

En remplaçant un trio paradoxal par un duo clairement ordonnable, la stratégie de la règle électorale escamote l'Effet Condorcet. Le paradoxe est levé. La confusion liée à une triade irrationnelle éventuelle est annulée par le recours à la platitude d'une dyade.

\subsection{UNE CONFRONTATION AMOUREUSE SHAKESPEARIENNE}

Une triade déséquilibrée, et a fortiori paradoxale, ne semble pas satisfaisante pour l'esprit. C'est un mathématicien de renom, pionnier de la Théorie des Graphes et de la relation de tournoi, Frank Harary, qui a montré comment une triade paradoxale fâcheuse a été éliminée par Shakespeare dans l'une de ses comédies : La Nuit des Rois [Harary, 1975]. L'illustre dramaturge met en scène un trio dont la relation intransitive entraîne un duc, une princesse et une jeune fille déguisée en page, dans un circuit amoureux sans issue ; le duc Orsino a demandé la main de la princesse Olivia ; mais celle-ci tombe amoureuse du page Viola (supposé être un jeune homme) qui lui-même (elle-même) s'est épris(e) du duc (cf. Figure 5a). Fort opportunément, ce réseau néfaste est redistribué grâce à l'intervention d'un jouvenceau séduisant, Sébastien, frère jumeau et sosie du page déguisé. Un amour réciproque réunira alors, d'une part la princesse et le jouvenceau dont elle s'est inopinément éprise (la ressemblance entre jumeaux étant parfaite), et d'autre part le duc et la fascinante jeune fille qui se cachait sous les habits masculins d'un page. L'intervention providentielle du quatrième personnage, Sébastien, brise le triangle maudit en créant deux couples-dyades désormais voués au bonheur (cf. Figure 5b).

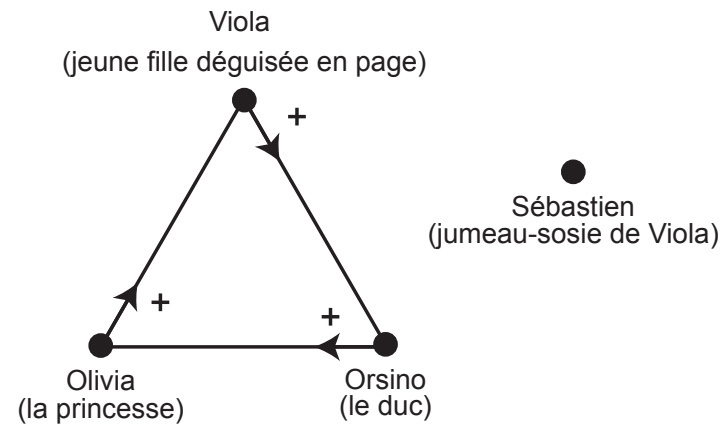

a) Début de pièce

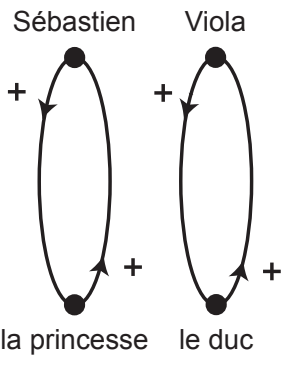

b) Fin de pièce

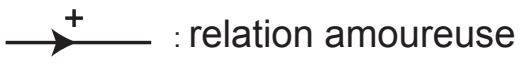

FIGURE 5. Élimination du paradoxe par l'intervention d'un providentiel quatrième personnage Le triplet intransitif se mue en deux couples harmonieux [La nuit des rois, W. Shakespeare - F. Harary]

De nombreuses œuvres littéraires (Racine, Corneille, Marivaux...) se prêteraient à une analyse en termes de graphes déséquilibrés, voire paradoxaux... leur dénouement évoluant quasi systématiquement vers la disparition ou l'adoucissement du paradoxe dont le «Va, je ne te hais point» de Chimène à Rodrigue fournit une éloquente illustration. Dans un chapitre de son ouvrage consacré aux triades, Th. Caplow analyse 
de façon ingénieuse les relations d'Hamlet avec son entourage, mais en recherchant constamment les coalitions gagnantes, ce qui revient à réduire les triades à des duels !

\subsection{UN JEU D’ AFFRONTEMENT AUX APPARENCES PARADOXALES : LE « SAGAMORE »}

Un exemple emprunté au domaine des jeux traditionnels va permettre d'illustrer comment des normes, apparemment ternaires et paradoxales, masquent en réalité une situation binaire dénuée de toute confusion.

Il s'agit d'un grand jeu de pleine nature appelé le «Sagamore », qui oppose deux équipes symétriques possédant la même répartition de leur douzaine de joueurs respectifs selon cinq rôles sociomoteurs : Sorcier, Chasseur, Sagamore, Sachem, Agent secret. Chacune des deux équipes choisit son camp dans un lieu caché qui sera le refuge du Sorcier. Tout joueur possède une «carte de rôle » confidentielle au départ, et va essayer de s'emparer de cartes adverses en évitant de se faire prendre la sienne. Au cours de ses déplacements, un joueur peut décider de défier un adversaire rencontré : dans ce cas, les deux joueurs doivent montrer leur carte, et celui dont le statut est dominant prend possession de la carte de l'autre. La Figure 6 indique la relation de dominance qui réglemente les affrontements de rôle à rôle. L'originalité de ce jeu tient au fait que les trois rôles majeurs sont en relation cyclique : le Sagamore a barre sur le Sachem, celui-ci sur l'Agent secret, et ce dernier en retour sur le Sagamore. Nous retrouvons ici la triade paradoxale repérée à Pierre-Feuille-Ciseaux, à Renard-PouleVipère, aux élections présidentielles et à la comédie de Shakespeare.

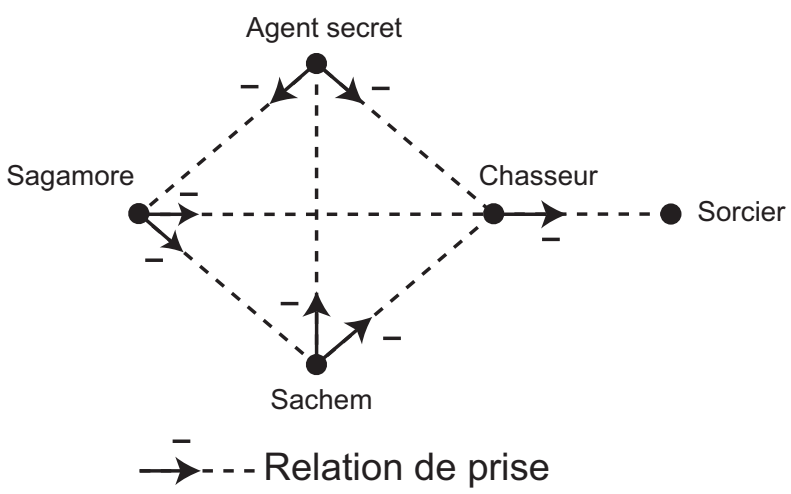

FIGURE 6. Graphe de dominance des rôles au Sagamore

On retrouve ici, dans un sous-graphe triangulaire intransitif, la triade paradoxale opposant de façon cyclique les trois rôles principaux du jeu : Sagamore, Sachem, Agent secret

Cependant, sur le terrain, tout comme à Pierre-Feuille-Ciseaux, mais à l'encontre de Renard-Poule-Vipère, les interactions des pratiquants n'offrent aucune ambiguïté : l'irrationalité des relations de rôles semble se dissoudre dans le caractère irréfutable et non négociable de la dominance entre rôles. Comment se fait-il que l'illogisme relationnel notoire détecté précédemment n'entraîne aucun désordre dans les affrontements ludiques?

En réalité, dans les représentations traditionnelles que l'on se fait de ces jeux, s'est installée une confusion entre les relations de rôles proprement dites et les relations des joueurs des deux équipes opposées qui assument ces rôles respectifs. Le Sagamore domine le Sachem, mais il s'agit du Sachem de l'équipe adverse ! De même pour les droits de prise du Sachem et de l'Agent secret, qui s'exercent uniquement à l'égard 
d'adversaires ! Si l'on dresse le graphe de la relation de domination qui relie les rôles des joueurs respectifs des deux équipes, on obtient un circuit de rivalité composé de six arcs négatifs (cf. Figure 7a). Ce 6-circuit est «équilibré » puisque son nombre d'arcs négatifs est pair [Heider, 1946 ; Harary et Cartwright, 1956 ; Flament, 1965]. Le graphe des rôles majeurs du Sagamore peut donc être bi-partitionné en deux sous-graphes séparés par des liaisons uniquement d'opposition définissant une «coupe » du graphe, de type antagoniste. Finalement, on se retrouve devant un jeu à deux super-joueurs et à somme nulle, c'est-à-dire devant un banal duel et non devant un jeu paradoxal (cf. Figure 7b).

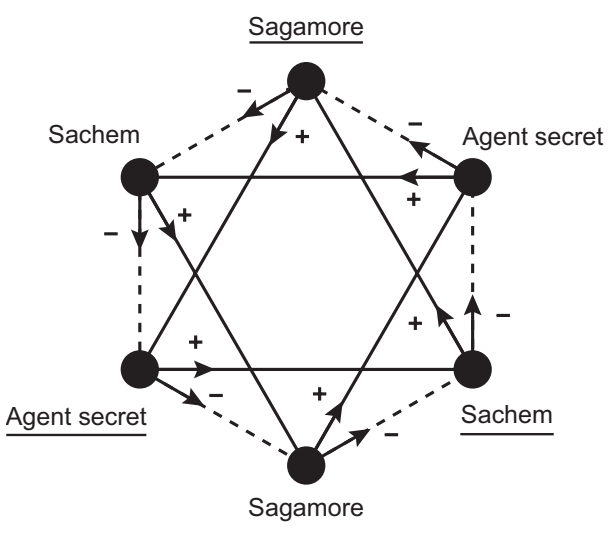

a) Graphe global, équilibré

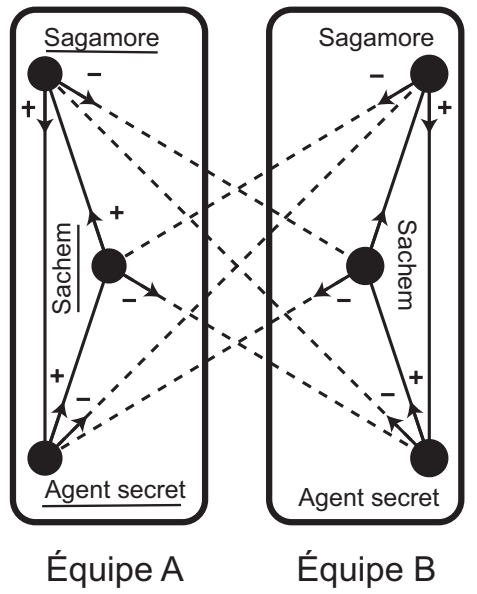

b) Le « même » graphe, sous forme de duel

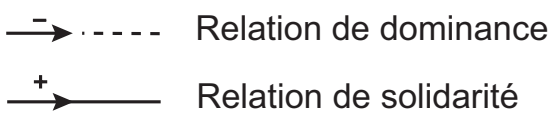

FIGURE 7. Le système des trois rôles majeurs du Sagamore

Bien que circulaire, la relation de dominance n'entraîne aucune incohérence : le cycle irrationnel n'apparaît pas car la liaison négative n'intervient que dans des paires de joueurs d'équipes opposées (sachant que la relation d'entraide ne s'exerce qu'au sein de chaque équipe)

En menant la même démarche auprès de «Pierre-Feuille-Ciseaux », on obtient un modèle identique selon la relation de rivalité $\mathrm{R}$ : une «coupe » strictement négative du graphe, qui illustre un duel dont les issues de chaque coup sont nettes et sans aucune incohérence (cf. Figure 8).

Il semble donc abusif d'associer «Pierre-Feuille-Ciseaux» à une situation cyclique qui créerait un imbroglio relationnel. Le jeu «Pierre-Feuille-Ciseaux » » est un duel classique, parfaitement logique, de type symétrique, dont les éléments se dominent circulairement de joueur à joueur de façon équilibrée. Si l'on ajoute un quatrième élément figuré par le Puits, le tournoi est encore affecté d'un circuit de type II qui modifie les probabilités de gain de chaque symbole, mais qui garde intacte la structure de duel dénuée de toute ambiguïté. 


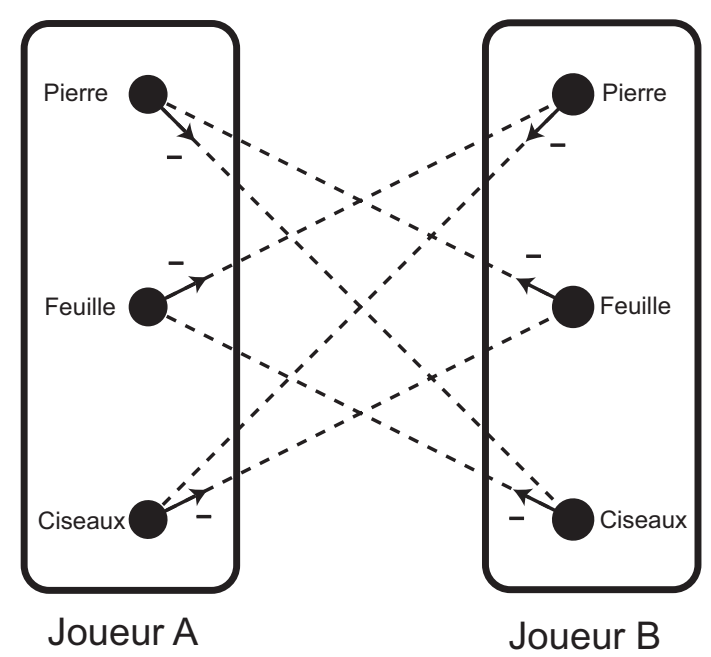

FIGURE 8. Relation de dominance à « Pierre-Feuille-Ciseaux » Elle définit une structure classique de jeu à deux joueurs et à somme nulle, et non une structure paradoxale

\subsection{LA TRIADE EN ÉQUIPES}

Le fait que ce soit des individus et non des équipes qui s'affrontent à «Pierre-FeuilleCiseaux » n'est pas un élément déterminant. Ainsi, dans le jeu «Nain, Géant, Magicien », ce sont deux équipes qui s'opposent de part et d'autre d'une ligne médiane qui les sépare. La règle stipule que les Nains dominent les Géants, que les Géants supplantent les Magiciens et que les Magiciens ferment la boucle en ayant barre sur les Nains. Le triangle paradoxal semble bel et bien être là.

Avant chaque coup du jeu, chacune des deux équipes décide en secret du rôle que tous ses membres vont représenter à l'unisson, sachant qu'au signal chaque équipe devra adopter immédiatement la posture corporelle correspondant au rôle choisi : accroupi pour les Nains, debout bras élevés à la verticale pour les Géants et debout bras écartés à l'horizontale pour les Magiciens. Dès le signal lancé, chaque joueur doit adopter sur le champ la posture de son équipe, identifier le rôle choisi par l'adversaire et décider à brûle-pourpoint de la conduite répondant au décodage du rapport des deux rôles affrontés : devenir poursuivant ou poursuivi jusqu'à la ligne-refuge du camp correspondant (ou rester neutre si les deux équipes ont choisi le même symbole). En fonction des règles de domination, tout joueur devient ou prédateur ou fuyard (ou neutre), l'objectif étant respectivement de toucher le poursuivi ou, à l'inverse, d'éviter cette capture. La dyade se substitue à la triade. Là encore, comme dans le cas de « Pierre-Feuille-Ciseaux », la relation cyclique qui symbolise ce jeu triadique s'évanouit au profit d'une liaison binaire de dominance, franche et d'une totale cohérence.

L'irrationalité de la dominance et de l'effet Condorcet n'est-elle qu'une apparence trompeuse qui se dissipe dans la réalité des comportements de terrain?

\section{LE PARADOXE, BEL ET BIEN}

L'observation des pratiquants du jeu des Trois camps dénote des phénomènes d'une tout autre nature, qui ne se réduisent pas à une stricte addition d'affrontements dyadiques. Sur le terrain de jeu, au bout de quelques phases de captures et de délivrances, les acteurs semblent quelque peu interloqués ; hésitants, ils ne savent plus 
s'il leur faut fuir devant leurs prédateurs ou s'élancer vers leurs victimes pré-désignées. Le fonctionnement du jeu est intriguant. Par exemple, si les Vipères capturent tous les Renards, dans un premier temps elles se réjouissent en étant convaincues qu'elles ont gagné ; cependant, dans les faits, elles s'aperçoivent qu'elles ne peuvent plus sortir de leur camp car les Poules, qui ne sont plus désormais menacées par les Renards annihilés, peuvent occuper le terrain en toute impunité. Qui donc a gagné ? Les Vipères qui ont éliminé toutes leurs victimes potentielles, ou les Poules qui se pavanent autour du camp des Vipères assiégées et immobilisées sans recours ?

$\mathrm{Au}$ cours de ce jeu, ce n'est pas la dyade qui prévaut, mais la triade. Quand le Renard capture la Poule, il se prive ipso facto de sa protectrice, seule susceptible de supprimer son adversaire direct, la Vipère. Dans toute liaison à deux, un tiers est omniprésent. Ce qui n'est pas le cas à «Pierre-Feuille-Ciseaux» au cours duquel toute interaction ne se joue qu'entre deux éléments. Tous les coups du jeu y sont indépendants en probabilité : à chaque fois, la partie recommence à zéro, avec une chance sur trois de gagner (et un risque sur trois de perdre); et les scores s'additionnent mécaniquement. À l'opposé, à «Renard-Poule-Vipère », les actions de jeu sont interdépendantes: d'une part, en délivrant les prisonniers, un partenaire modifie brutalement le rapport des forces (ici, les stocks de joueurs et de points ne sont pas infinis comme à «Pierre-Feuille-Ciseaux »); d'autre part, de façon plus subtile, en capturant la Poule, le Renard sauve la Vipère, cette Vipère qui est pourtant son épée de Damoclès!

Dans le triangle paradoxal où la relation $R$ de domination crée une rivalité circulaire, chaque joueur protège donc son prédateur (cf. Figure 9 ). On est en présence d'une relation indirecte faisant intervenir un étrange intermédiaire: il s'agit d'une relation « relayée » de solidarité $S$ ', telle que la victime protège son bourreau !

Sachant que $r$ (Renard), $p$ (Poule) et $v$ (Vipère) appartiennent respectivement aux trois camps en présence composant l'ensemble $E$ des joueurs, il vient :

$$
\forall r, p, v \in E: \quad(r, p) \in R \quad \text { et } \quad(p, v) \in R \quad \Rightarrow \quad(r, v) \in S,
$$

De façon paradoxale, cette relation de solidarité $S^{\prime}$ ' «transitive » la relation $\mathrm{R}$ de rivalité (cf. Figure 9). Pour bien mettre en évidence cette intervention « intermédiaire », on peut symboliser cette liaison « relayée » par la relation ternaire de médiation $\mathrm{M}$ qui présente l'avantage, pour notre problématique, de réinterpréter une relation binaire en une relation ternaire. Il vient :

$$
(r, p) \in R \quad \text { et } \quad(p, v) \in R \quad \Rightarrow \quad(r, p, v) \in M
$$

ce qui entraîne :

$$
(r, p, v) \in M \quad \Rightarrow \quad(r, v) \in S,
$$

et identiquement :

$$
\begin{aligned}
& (p, v, r) \in M \quad \Rightarrow \quad(p, r) \in S \\
& (v, r, p) \in M \quad \Rightarrow \quad(v, p) \in S
\end{aligned}
$$

Ces caractéristiques provoquent les chocs relationnels contradictoires suivants, pétris d'incohérence au sein de chaque paire d'adversaires :

$$
\begin{array}{lll}
(v, r) \in R & \text { et } & (r, v) \in S, \\
(r, p) \in R & \text { et } & (p, r) \in S, \\
(p, v) \in R & \text { et } & (v, p) \in S
\end{array}
$$




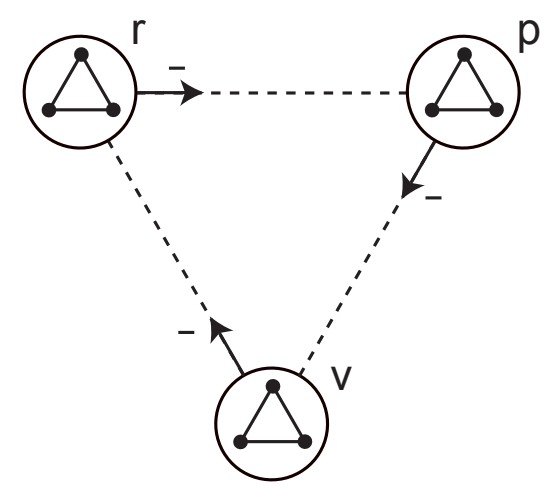

a) Graphe cyclique de la relation «R» de dominance

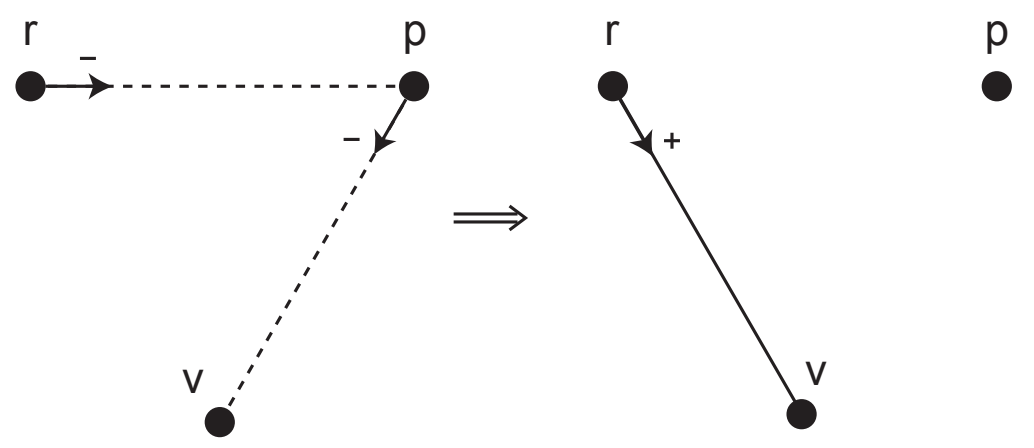

b) Conséquence contradictoire pour chaque paire. Ici, $(r, v) \in S^{\prime}$ et $(v, r) \in R$

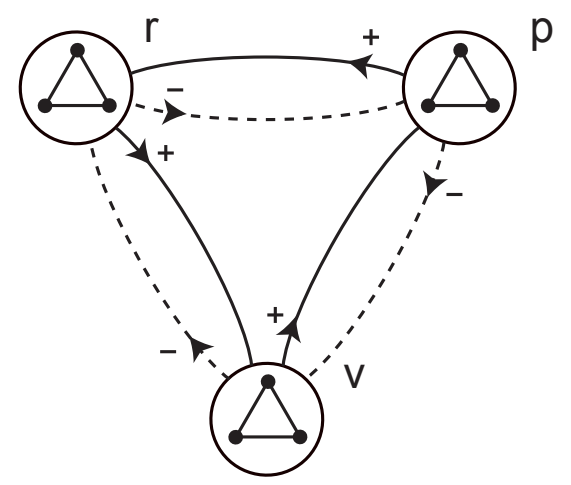

c) Graphe paradoxal résultant

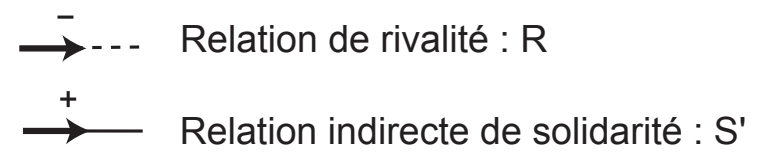

FIGURE 9. La triade paradoxale de « Renard-Poule-Vipère »

La relation $R$ de rivalité engendre une relation « relayée » $S$ ' de solidarité, qui est l'inverse de $R$ :

la vipère $« v »$ a pour objectif de s'emparer du Renard $« r »$, mais ce dernier est précisément celui qui la protège (en prenant la poule « $p »$ qui menace $« v »)$ !

L'ambivalence est ainsi au cœur des interactions ludiques 
La relation binaire $S$ ' est donc l'inverse de la relation $R$ : la relation ternaire de solidarité $M$ est bâtie sur une relation binaire de rivalité ! La conclusion s'impose : les deux membres de toute paire de joueurs d'équipes différentes sont à la fois adversaires et partenaires! Cette ambivalence, déconcertante, est au cœur des conduites des pratiquants d'un jeu paradoxal et, à vrai dire, souvent difficile à gérer dans l'ensemble de ses conséquences.

C'est là que gît le paradoxe : pour vaincre, le Renard doit prendre la Poule, mais en prenant la Poule il se prive de son sauveur; en faisant ce qu'il faut pour gagner, il concourt à sa perte. Le joueur est donc enserré dans l'étau d'une double obligation contradictoire. Il est pris entre deux injonctions opposées qui renvoient l'une à l'autre. Ce type de situation, à l'origine de nombreux dysfonctionnements de la vie quotidienne, a été longuement analysé par le psycho-sociologue Gregory Bateson qui l'a qualifié de « double contrainte » [Bateson, 1977]. Celle-ci, ludique et non pathologique, est bel et bien constitutive de la triade paradoxale de «Renard-Poule-Vipère ». Elle est également interprétable en terme «d'effet pervers» selon l'analyse du sociologue Raymond Boudon. En effet, c'est intentionnellement que le Renard s'empare de la Poule, entraînant ainsi des conséquences non intentionnelles [Boudon, 1977].

\section{MORPHOLOGIE TRIADIQUE}

Il est souvent affirmé que les jeux traditionnels sont des «petits » jeux puérils, voire simplets, ne sollicitant que des qualités superficielles des participants. Étonnant préjugé affectant des pratiques dont la complexité relationnelle tranche, face à l'évidente simplicité dichotomique du réseau des sports collectifs pourtant réputés supérieurs.

Considérons une partie de «Renard-Poule-Vipère » mettant en scène $\mathrm{N}$ joueurs répartis en trois équipes de $n$ membres chacune, dont le réseau d'interactions est assuré par une relation négative de capture et une relation positive de soutien et de délivrance. Quelle est la morphologie des différentes triades en jeu ? Afin de se donner un aperçu des situations auxquelles les joueurs doivent faire face, peut-on dénombrer les divers types de triades dans lesquelles sont impliqués, d'une part l'ensemble des participants, d'autre part un joueur particulier?

Soit 21 joueurs répartis en trois équipes de 7 éléments chacune. La configuration globale admet un total de $N(N-1)(N-2) / 6=1330$ triades. Celles-ci se répartissent en trois grands types (cf. Figure 10) :

- $1^{\text {er }}$ type : les triades internes à chaque équipe, totalement positives et bien entendu « équilibrées » (105 cas pour l'ensemble, et 35 pour un joueur).

- $2^{\mathrm{e}}$ type : les triades reliant deux équipes, possédant deux arcs négatifs, équilibrées ; elles admettent 4 sous-types ( 882 cas pour l'ensemble, et 126 pour un joueur).

- $3^{\mathrm{e}}$ type : les triades reliant les trois équipes, possédant 3 arcs négatifs en circuit, non équilibrées, paradoxales (343 cas pour l'ensemble, et 49 pour un joueur).

Le paysage triadique est particulièrement fourni et bariolé. Bien entendu, le joueur n'a pas à se soucier d'un tel dénombrement! En revanche, il lui faut sur le champ identifier les différents types de triades changeantes dans lesquelles il est à tout instant impliqué. Celles qui posent de réelles difficultés de décision et d'action, ce sont évidemment les triades du $3^{\mathrm{e}}$ type, fortes de plusieurs dizaines, chacune exerçant concrètement des contraintes particulières plus ou moins menaçantes selon l'âge, le genre et les capacités des protagonistes. Le décodage de la situation requiert des appréciations judicieuses et des anticipations motrices habiles qui se succèdent de façon 
continue au cours d'une action réciproque dépendante des déplacements des joueurs. La difficulté est accrue par le fait que les triades s'entrelacent en formant des configurations complexes, sollicitant souvent 4,5 ou 6 joueurs au contact.

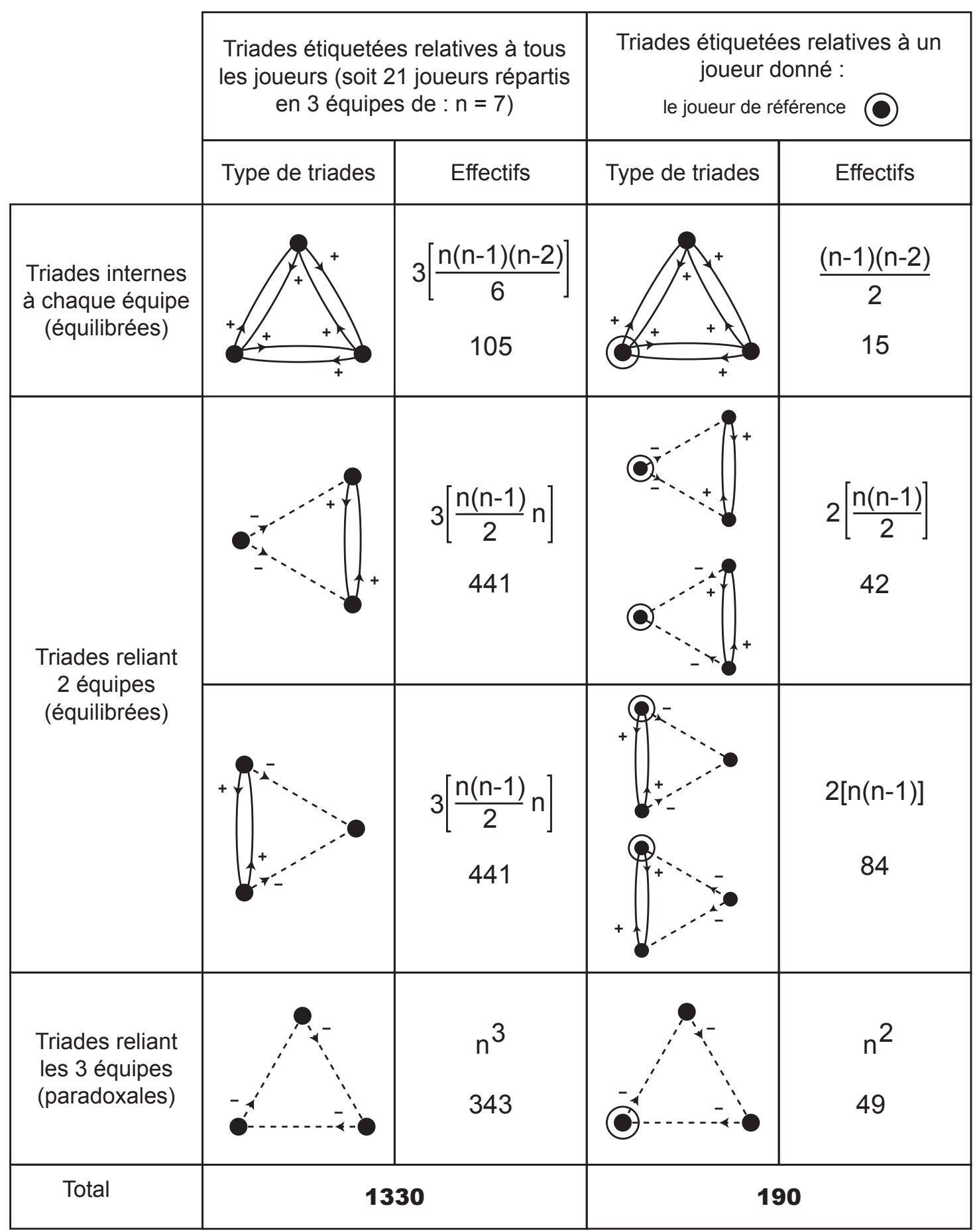

FIGURE 10. Dénombrement des triades du jeu «Renard-Poule-Vipère » (soit 3 équipes de 7 joueurs chacune)

Le champ des possibles est particulièrement vaste et oblige le joueur à faire face à une foule de situations fort différentes 
Deux types de facteurs, notamment, influencent les confrontations : d'une part les ressources physiques des pratiquants liées aux distances de course qui les séparent, et d'autre part les intentions prêtées aux autres pratiquants dont on ne sait s'ils vont se comporter en prédateurs ou en complices provisoires. Sur le plan relationnel des stratégies adoptées, les acteurs peuvent négocier et nouer des alliances. Par empathie, chacun va essayer de deviner ce que projettent les autres. Un Renard proposera à une Poule de l'accompagner afin qu'elle le protège contre les Vipères, de telle sorte qu'il puisse délivrer ses compagnons prisonniers des Vipères ; et, en retour, il lui promettra de ne pas la capturer. Qu'en sera-t-il ? Respect de la promesse ou trahison finale ? La règle du jeu donne toute liberté au joueur pour tenir parole ou pour rompre inopinément l'accord passé. Les conduites des joueurs, belliqueuses ou doucereuses, vont être fondées sur la confiance et la loyauté réciproques. "Je te soutiens, moi non plus »: équilibre fragile, inéluctablement condamné à la trahison, car comment le Renard pourrait-il réussir si, en dernier ressort, il ne prend pas la Poule ? La tension qui règne entre deux adversaires est toujours médiée par un troisième larron ; mais, dans ce circuit triadique, chacun peut devenir le troisième larron des deux autres, ce qui provoque une situation inextricable. Aussi apparaît-il indispensable de distinguer la situation « PierreFeuille-Ciseaux » composée d'une succession de duels dyadiques tranchés, de la situation «Renard-Poule-Vipère » entraînée dans le tourbillon d'une circularité triadique inachevable.

\section{UN TRIANGLE MAUDIT?}

\subsection{UNE UNITÉ FONDATRICE}

Le jeu «Pierre-Feuille-Ciseaux» évoqué par J.-P. Delahaye pourrait paraître bien anodin, limité à l'anecdote d'un divertissement enfantin. En réalité, en mettant la triade au centre des problèmes d'interaction, l'auteur a ravivé un thème jugé crucial par de nombreux sociologues. On sait que l'un des pionniers de la sociologie, Georg Simmel, avait insisté sur l'analyse microsociologique de l'action réciproque vécue dans les multiples interactions de la vie quotidienne. Dans cette lignée, Théodore Caplow a explicitement approfondi les liaisons qui fondent les configurations à trois acteurs. Dans son ouvrage au titre évocateur Deux contre un, il identifie huit types de triades caractéristiques en fonction des rapports de force provoqués par le poids de chaque élément et par les différentes possibilités de coalitions qui en résultent. Son propos est sans équivoque : «les triades, écrit-il, sont les matériaux de base à partir desquels se construisent toutes les organisations sociales »[Caplow, 1971]. Sachant que les membres d'une triade peuvent être des individus ou des collectivités, il applique résolument son point de vue aux organisations qu'il qualifie «de grande envergure » : usines, agences, entreprises, administrations, partis politiques...

Plus récemment, Michel Forsé a repris le flambeau ; il souligne la fécondité de la conception de Simmel et rappelle que, pour ce sociologue, « la figure fondamentale de l'interaction est à rechercher dans la triade » [Forsé, 1994]. En affirmant qu'une triade ne se réduit pas à une somme de dyades, il confère à cette mini-configuration une originalité fondatrice, que nous avons retrouvée dans le jeu des Trois camps. Insistant sur le fait que «La plus petite unité pertinente est la triade », M. Forsé redore le blason de ce micro-réseau basique souvent laissé dans l'ombre par les théories macrosociologiques. Déjà, les recherches sociométriques ont largement mis en évidence le rôle du tissu relationnel de base [Moreno, 1934]. Dans cette perspective, une analyse en terme de triade a permis de dépasser l'intimité des dyades sociométriques pour 
introduire une dimension résolument sociale sous forme d'un troisième intervenant: ainsi ont été présentés les «sociogrammes triadiques» de type individuel et de type collectif [Parlebas, 1992].

\subsection{L'EXCLUSION DU PARADOXE}

Les analyses de Condorcet, qui reprenaient des recherches de Borda datant de 1780, ont été mises sous le boisseau pendant plus d'un siècle et demi. Ce n'est que vers 1950 que Black et Arrow en ont redécouvert les principes, symbolisés par le «théorème de l'impossibilité »d'Arrow. À une époque qui s'affirmait révolutionnaire, ces résultats étaient gênants : ils menaçaient l'expression de la volonté générale. Ils ne pouvaient être reçus sereinement à la fin du XVIII ${ }^{\mathrm{e}}$ siècle, dans un temps où les nations souhaitaient se débarrasser des pouvoirs monarchiques et despotiques au profit des représentants élus par des majorités démocratiques. Le paradoxe de Condorcet était ainsi perçu comme le trio maudit qui faisait obstacle à l'expression libre et cohérente d'une majorité de citoyens.

Le pas de la reconnaissance du paradoxe semble difficile à franchir. Même un innovateur tel Th. Caplow ne réussit pas à considérer la triade comme une unité de base originale pouvant entraîner par elle-même une ambivalence fondatrice. Le caractère irrationnel de l'effet Condorcet lui paraît rédhibitoire. Tout en reconnaissant la présence de phénomènes d'ambivalence, il en réduit l'impact en déclarant «incompatibles » les coalitions engagées dans des affrontements autres que strictement binaires. Aussi en vient-il à nier la possibilité de liaisons ambivalentes entre les protagonistes d'une même activité collective: "Des éléments pourront être partenaires dans une coalition et adversaires dans une autre, affirme-t-il, mais pas à l'intérieur du même système d'action» [Caplow, 1971]. Cela ne se peut «absolument pas », insiste-t-il, en choisissant comme exemples le bridge et le tennis qui permettent respectivement que des partenaires deviennent adversaires mais uniquement au cours de parties successives différentes. Et il revient sur cette idée-force en martelant qu' « en fin de compte toute bataille se joue entre deux armées, et deux seulement». Or, ce sont précisément les prétendues incompatibilités de Caplow qui dessinent l'armature de la triade paradoxale rejetée comme un triangle maudit.

C'est la même réaction d'exclusion de l'irrationnel qu'a signifiée Henri Troyat en donnant pour titre à l'un de ses romans «La pierre, la feuille et les ciseaux », alors qu'à aucun moment de son récit, il ne fait allusion à cette pratique ludique. La description précise qu'il propose de ce jeu en épigraphe de son livre, et que nous avons retranscrite en tête de cet article, souligne les affrontements sévères qui opposent les trois personnages marquants de son roman. Cet éminent membre de l'Académie Française ne se fourvoie-t-il pas dans la description qu'il offre métaphoriquement des conflits évoqués, en proposant le modèle explicatif tranchant de «Pierre-Feuille-Ciseaux » manifestement inadéquat? Les personnages d'H. Troyat vivent en réalité une relation circulaire, dans une circularité spécieuse, apparemment maudite, qui est à la source des superbes inconstances de leurs actions réciproques relevant davantage du paradoxe triadique que du duel dyadique.

La conclusion que l'on peut tirer des différents cas évoqués est fortement marquée: dans les multiples situations où il se produit, le circuit triadique est habituellement mal accepté et fait figure de trio maudit. Ainsi que nous l'avons observé, plusieurs façons de l'éliminer ont été adoptées, notamment :

- supprimer l'un des trois éléments de la triade en ne conservant qu'une seule dyade (exemple de l'élection présidentielle). 
- ajouter un quatrième élément qui transforme la triade intempestive en deux dyades harmonieuses (exemple de la Nuit des Rois).

- regrouper deux éléments en un seul, formant ainsi une alliance qui transforme la triade en une dyade unique (exemple des travaux de Th. Caplow).

- supprimer le circuit triangulaire perturbant au profit d'une juxtaposition de trois dyades indépendantes (exemple de «Renard-Poule-Vipère » remplacé par «PierreFeuille-Ciseaux $\gg$ ).

$\mathrm{Au}$ cours d'une partie de «Renard-Poule-Vipère », les enfants jeunes sont décontenancés par les conséquences relationnelles du paradoxe, et proposent souvent de changer les règles : ils demandent que l'équipe ayant, la première, capturé tous ses adversaires (ou un nombre arrêté d'entre eux) soit déclarée gagnante. Cette règle d'arrêt brise l'indétermination. Dans le même esprit, de jeunes enfants s'adonnant à la Balle Assise, perturbés d'être de façon contradictoire à la fois partenaire et adversaire de chacun, demandent parfois que les joueurs soient répartis dans deux équipes opposées, de telle sorte qu'un tel duel d'équipes supprime l'ambivalence fondatrice du jeu.

En revanche, les préadolescents, les adolescents et les adultes se livrent avec enthousiasme aux subtilités des échanges de «Renard-Poule-Vipère » et des jeux paradoxaux en général (à la suite, il est vrai, d'un étonnement et d'un flottement en début de partie). Après coup, les joueurs déclarent être enchantés de cette expérience inhabituelle, de ces échanges teintés de ruse et d'humour, de ces stratégies de conciliabules et de provocations. Avec le risque permanent d'un brusque renversement des situations, qui désarçonne même les vieux routiers des jeux. Cette aventure ludique, reposant sur une adaptabilité périlleuse, est ressentie comme une expérience originale et plaisante, renouvelant le vécu du lien social.

\subsection{UNE RÈGLE QUI DÉRÈGLE}

Ce refus de la dissonance paradoxale qui hante les représentations courantes, ne doit pas trop étonner. Etant donné, constate R. Boudon, que les effets pervers « représentent une des causes fondamentales des déséquilibres sociaux et du changement social », les agents sociaux vont tout faire pour les éliminer [Boudon, 1977]. Le cas du sport en offre une illustration spectaculaire. Le patrimoine séculaire des jeux traditionnels possède un effectif non négligeable de pratiques suscitant, à des degrés divers, des ambivalences paradoxales: certains prescrivent ouvertement le paradoxe (Renard-Poule-Vipère), d'autres en provoquent l'émergence moins directement mais de façon inéluctable (la Balle assise, la Galine, les Quatre coins, la Balle empoisonnée...), d'autres enfin en favorisent l'apparition sous une forme plus discrète (Accroche-décroche, le Gouret, la Porte, Chat coupé...). Or, dans ce domaine des activités ludomotrices, le constat est limpide : aucun jeu physique institutionnalisé, c'est-à-dire aucun sport, n'accepte cette incohérence. L'effet pervers est impérativement rejeté.

Les règles du sport peuvent être interprétées comme le système de contraintes imposé par l'institution en vue d'éliminer les effets pervers désorganisants, tout en exaltant le modèle ordonné du duel. Là encore, on impose "Pierre-Feuille-Ciseaux » aux dépens de «Renard-Poule-Vipère ». L’indiscipline individuelle et la discordance paradoxale terniraient comme une tache le blason sportif. Le sport ne veut pas d'une règle qui dérègle. 


\section{LA THÉORIE DES JEUX DÉJOUÉE?}

Au cours des péripéties ludiques, le pratiquant de «Renard-Poule-Vipère », et plus généralement d'un jeu paradoxal, peut prendre de multiples initiatives; les contraintes du règlement l'autorisent par exemple à capturer à son gré tel joueur plutôt que tel autre, à esquiver telle attaque ou à délivrer préférentiellement tel compagnon. Les comportements du terrain font la part belle aux foucades des pratiquants qui agissent parfois de façon apparemment incohérente, en nouant une alliance improbable, en favorisant la prise d'un partenaire ou en se laissant capturer facilement. D'une façon générale, les jeux paradoxaux dont l'ambivalence est à fleur de comportement multiplient les interactions subversives qui semblent des défis fantaisistes et gratuits, apparemment illogiques dans le contexte donné.

L'observation et l'analyse précise des actions de jeu révèlent que les pratiquants n'adoptent pas toujours des conduites utilitaristes strictement rationnelles, en vue de maximiser des réussites ou des «gains » évaluables sur le mode standard de l'économie. Ces joueurs obéissent à des motivations plus subtiles et moins comptables, se plaisent à la provocation, font preuve d'humour, agressent subitement le compagnon qu'ils protégeaient la minute précédente, s'adonnent parfois à la coopération aux dépens de leur intérêt immédiat. Une enquête approfondie auprès d'enfants d'école maternelle et d'athlètes olympiques s'adonnant au jeu des Quatre coins, a montré que ces joueurs ne se comportent pas de façon logique : ils prennent des risques inconsidérés alors que leur réussite était assurée, ils adoptent des itinéraires qui les font perdre immanquablement, ils se laissent prendre un coin qui était à leur portée... [Parlebas, 1974]. Le traitement des déplacements des pratiquants et l'analyse de leurs entretiens post-expérimentaux ont permis de mieux comprendre les motifs de leur conduite. La logique du joueur n'épouse pas nécessairement la froide rationalité «économique». Ainsi que le note R. Boudon : « tout comportement a en principe ses raisons ou ses bonnes raisons » [Boudon, 2003] ; et le joueur paradoxal a souvent de bonnes raisons que la raison économique ne connaît guère. La rationalité n'est pas toujours là où on l'attend !

C'est sans doute la prise en compte d'un point de vue trop strictement quantitativiste lié aux forces brutes des coalitions, qui a conduit Th. Caplow à ignorer la triade intransitive. Et c'est en grande partie une telle conception rigide, fondée sur une hypothèse de maximisation des gains, qui a rendu réticents de nombreux sociologues à l'égard de la Théorie des Jeux. Sans doute influencée par le rôle de l'économétricien Oskar Morgenstern lors de la co-rédaction avec John Von Neumann de l'ouvrage-phare Théorie des Jeux et comportement économique, cette orientation a donné de la Théorie des Jeux une image trop formaliste, prisonnière de calculs sophistiqués qui ne pénètrent pas la subjectivité des représentations des acteurs. Cette dérive n'a pas échappé à J.-P. Delahaye avançant qu' «il faut renoncer à considérer qu'il n'y a en nous qu'un agent économique purement rationnel cherchant sans faiblir à optimiser son profit » [Delahaye, 2010]. Le sociologue peut se saisir avec grand profit de la démarche et des modèles de la Théorie des Jeux, mais tout en gardant ses propres formes habituelles d'explication et de compréhension; aucune fatalité ne l'oblige à adopter le point de vue utilitariste, strictement économiste. La Théorie des Jeux doit laisser du « jeu » dans le jeu !

L'exemple proposé par J.-P. Delahaye dans une démarche interdisciplinaire, est particulièrement évocateur. Le cas des lézards californiens dont les interactions dépendent circulairement les unes des autres, est du côté de "Renard-Poule-Vipère ». Complétant cette illustration par d'autres exemples empruntés à l'éthologie et à la biochimie, s'appuyant sur d'imposantes simulations informatiques, l'auteur en arrive à une conclusion étonnante fondée sur le rôle de la triade circulaire : « la coexistence des 
trois symboles, écrit-il, est sans doute une explication du maintien de la diversité de certaines situations biologiques » [Delahaye, 2008]. Loin d'être stérile et perturbatrice, la circularité triadique deviendrait ici un facteur notoire de la biodiversité animale.

Cette hypothèse est audacieuse et importante, notamment si on la transpose - avec les précautions d'usage - dans le champ de la socialisation, telle que la conçoit G. Simmel par exemple. Quelles pratiques ludo-corporelles convient-il de proposer aux enfants pour qu'ils acquièrent durablement une sociabilité souple et ouverte ? Les duels sportifs sont intéressants, mais leur brutale sanction de dominance risque d'être ressentie comme «excluante»; en revanche, de nombreux jeux traditionnels paradoxaux, proposent une compétition davantage perçue comme «partageante». L'aspect subversif du jeu paradoxal provoque un choc qui desserre les contraintes et qui offre de nouvelles façons d'interagir.

À côté du modèle stimulant mais séparateur imposé par le duel et les coalitions gagnantes du sport, un modèle acceptant la coopération intermittente, la fantaisie et le « double jeu » semble nécessaire. C'est là une piste intéressante qui rompt avec le cadre unique du modèle compétitif du sport, tant sur le plan sociologique qu'éducatif. La flexibilité des interactions ludiques et l'ambivalence qui hante les interactions paradoxales apparaissent comme la promesse d'une ludo-diversité favorable à une sociabilité ouverte et épanouie. Si la relation paradoxale favorise le maintien de la biodiversité, est-ce faire preuve d'impudence de penser qu'elle favorise aussi le maintien de la socio-diversité ?

\section{BIBLIOGRAPHIE}

ARROW J.K. (1951), Social choice and individual value, New York, John Wiley \& Sons. Choix collectif et préférences individuelles, trad. par groupe de traductions économiques de l'Université de Montpellier, Paris, Calmann-Lévy, 1974.

BARBUT M. (1959), «Quelques aspects mathématiques de la décision rationnelle », Les Temps Modernes 164, Paris, p. 725-745.

BATTEAU P. (1979), Essays in the theory of collective choice, Northwestern University (Illinois).

BATESON G. (1977), Vers une écologie de l'esprit, Tome 1, Paris, Éditions du Seuil.

BOUDON R. (1977), Effets pervers et ordre social, Paris, Presses Universitaires de France.

BOUDON R. (2003), Raison, bonnes raisons, Paris, Presses Universitaires de France.

CAPLOW TH. (1971), Deux contre un. Les coalitions dans les triades, Paris, Librairie Armand Colin.

CONDORCET J.-A.-N. (1785), Essai sur l'application de l'analyse à la probabilité des décisions rendues à la pluralité des voix, Paris, Imprimerie royale.

DELAHAYE J.-P. (2008), « Logique et calcul. Pierre, feuille, ciseaux », Pour la science 36, p. 90-95.

DELAHAYE J.-P. (2010), Jeux finis et infinis, Paris, Éditions du Seuil.

FLAMENT C. (1965), Théorie des graphes et structures sociales, Paris, Gauthier-Villars.

FORSE M. (2002), «Les réseaux sociaux chez Simmel : les fondements d'un modèle individualiste et structural », sous la dir. de L. Deroche-Gurcel et P. Watier, La Sociologie de Georg Simmel (1908). Éléments actuels de modélisation sociale, Paris, Presses Universitaires de France, p. 63-109.

GOLDBERG J. (1998), Les sociétés animales, Lausanne-Paris, Delachaux et Niestlé SA.

GUILBAUD G.-TH. (1952), «Les théories de l'intérêt général et le problème logique de l'agrégation », Économie appliquée 5, repris dans Éléments de la théorie des Jeux, Paris, Dunod, 1968.

HARARY F. (1975), « Le graphe de la Nuit des Rois », Mathématiques et Sciences humaines 51, p. 77-80. 
HUDRY O. (1996), «Tournois et analyse des préférences ordinales », Mathématiques, Informatique et Sciences humaines 133 , p. 5-6.

HUDRY O. (2003), «Votes et paradoxes: les élections ne sont pas monotones », Mathématiques et Sciences humaines, $\mathrm{n}^{\circ}$ spécial : «Théorie du choix social : cinquantenaires »163, p. 9-39.

MONJARDET B. (1973), «Avant-propos » au n spécial «Opinions et scrutins : analyse mathématique », Mathématiques et Sciences humaines 43, p. 5-6.

MONJARDET B. (1973), «Tournois et ordres médians pour une opinion», $\mathrm{n}^{\circ}$ spécial «Opinions et scrutins : analyse mathématique », Mathématiques et Sciences humaines 43, p. 55-70.

MONJARDET B., HUDRY O. (2003), «Liminaires» au $\mathrm{n}^{\circ}$ spécial «Théorie du choix social: cinquantenaires », Mathématiques et Sciences humaines 163, p. 5-7.

MORENO J.-L. (1934), Fondements de la sociométrie, Paris, Presses Universitaires de France [2 édition 1970].

PARLEBAS P. (1971), «Effet Condorcet et dynamique sociométrique. 1. L'ordre de préférence au niveau individuel », Mathématiques et Sciences humaines 36, p. 5-31.

PARLEBAS P. (1972), «Effet Condorcet et dynamique sociométrique. 2. Incohérences rationnelles et cohésions groupales », Mathématiques et Sciences humaines 36, p. 37-67.

PARLEBAS P. (1992), Sociométrie, réseaux et communication, Paris, Presses Universitaires de France.

PARlebAS P. (1974), « Analyse mathématique élémentaire d'un jeu sportif », Mathématiques et Sciences humaines 47, p. 5-35.

TROYAT H. (1972), La pierre, la feuille et les ciseaux, Paris, Flammarion. 